\title{
Recognition of Oil and Gas Reservoir Space Based on Deep Learning
}

\author{
Shasha Yang ${ }^{1, a}$, Anjie Jin ${ }^{1}$ and Yongfu $\mathrm{Xu}^{2}$ \\ ${ }^{1}$ Shaanxi Key Laboratory of Safety and Durability of Concrete Structures, XiJing University, Xi'an, Shaanxi, 710123, China \\ ${ }^{2}$ Longdong Natural Gas Project Department, Petro China Changqing Oilfield, Qingyang, Gansu,745100, China
}

\begin{abstract}
The identification of oil and gas reservoir space is of great significance to oil and gas exploration. Deep learning technology represented by convolutional neural network is currently the most widely used artificial intelligence method in the field of image recognition. Using convolutional neural networks to identify the type and content of the reservoir space can not only ensure the objectivity and accuracy of the results, but also reduce labor costs and improve work efficiency. It has achieved good results in the identification of the reservoir space of the Chang 6 oil-bearing group in the Ordos Basin, which has a certain promotion significance.
\end{abstract}

\section{Introduction}

Oil and gas reservoir space, as the name implies, is the space for storing oil and natural gas. There are many differences in the types of reservoir space in different lithological reservoirs. For example, the reservoir space in sandstone reservoirs are mainly divided into three types, primary pores, secondary pores and microfractures. Under the same conditions of oil and gas reservoirs, the exploration of reservoir space has become the most direct and effective means of searching for oil and gas. From the content point of view, under normal circumstances, the higher the content of the reservoir space in the reservoir, the more oil and gas are stored. From the perspective of types, to determine the main types of reservoir space, one can reversely find the reservoir space by analyzing its formation mechanism and main controlling factors [1-3]. In short, the identification of the type and content of the reservoir space is extremely critical for oil and gas exploration.

The conventional means of identifying the type and content of reservoir space is to identify the type of reservoir space and calculate the content of ordinary rock thin slices and cast thin slices through electron microscope scanning or manual observation. This traditional method is not only extremely inefficient, but also lacks objectivity in the conclusions drawn due to excessive reliance on the experience of experimenters. To solve this problem, Jiubo proposed to use Matlab software to automatically characterize the pores of tight sandstone reservoirs[4], which effectively improved the efficiency and objectivity of the characterization of the reservoir space content, but did not identify the pore types. Qi Minghui proposed to use JMicro Vision image analysis software to analyze the scanning electron microscope images of shale to determine the pore type, content and other parameters[5]. However, this method requires a series of operations such as sketching and setting scales manually according to the shape of different reservoir space. The process is complicated and the objectivity cannot be guaranteed due to excessive manual intervention.

The ultra-high-speed development of computer and Internet technology, and the revolution brought about by machine learning have brought a new dawn to the development of artificial intelligence. Machine learning includes shallow learning [6] and deep learning [7].The revolution in machine learning comes from the research and development of biological neural networks [8]. People use computer neural networks to simulate biological neural networks to establish relevant scientific models, and have made breakthroughs in image recognition, speech recognition and artificial intelligence. The deep learning stage of machine learning can be traced back to 2006. At that time, neural network expert Hinton et al. published papers on deep learning and Boltzmann machines in the journal "Science", and proposed multiple hidden Layer to reinforce the point of view of artificial neural networks. In the article, Hinton puts forward two views that have contributed greatly to machine learning. First, more depth can be that the machine model can learn more features, and can better characterize things, which can improve the accuracy of recognition. Second, multiple hidden layers can transmit information, each hidden layer can be trained separately, and each layer adopts independent learning, which solves the embarrassing situation of manual training. The deep machine learning model is established by simulating the human brain. When humans process external information, they have to go through multiple levels of abstraction and transmission. Deep learning also establishes multiple levels of processing layers,

a Corresponding author: 20190100@xijing.edu.cn 
passing through low-level to high-level transmission and Abstraction, the conversion of low-level features to highlevel features, high-level features can better represent things. The deep learning model overcomes the problem of manually selecting features. In image processing, there are many difficult features such as HOG, LBP, and SIFT. The deep learning model can be automatically learned through the underlying features, reflecting the advantages of the deep learning model . The deep learning model has the ability to learn independently, does not require manual intervention, and shows good performance in the face of massive image data. The deep learning model does not use artificial rules to constrain the entire system model, so that the model has a more open state, and can be re-learned independently through the previously learned rules to enhance the model, which is suitable for massive image recognition. Deep learning models can develop rapidly, and breakthroughs have been made in many fields. In fact, there are many prerequisites. First, massive pictures are used as training data. Second, the construction of parallel platforms, the rapid development of computer hardware, and the development of GPUs, Multi-core integration provides a powerful hardware processing platform. Thirdly, the input of major universities and major Internet companies has improved the design and training methods of deep learning models. After decades of hard work, deep machine learning has achieved excellent results, produced excellent models such as convolutional neural networks, and made great contributions to image recognition.

Based on the above analysis, this paper proposes a method for identifying the type and content of oil and gas reservoir space based on deep learning technology. Deep learning technology represented by convolutional neural network is currently the most widely used artificial intelligence method in the field of image recognition. Using deep learning technology to identify the type and content of the reservoir space can not only ensure the objectivity and accuracy of the results, but also reduce labor costs and improve work efficiency.

\section{Oil and Gas Reservoir Space Recognition Technology Based on Convolutional Neural Network}

Convolutional Neural Networks (CNN) is a type of Feedforward Neural Networks that includes convolution calculations and has a deep structure. It is one of the representative algorithms of deep learning $[9,10]$. The Research Convolutional Neural Network has the ability to characterize learning, and can classify the input information according to its hierarchical structure, so they are also called "shift-invariant artificial neural networks (Shift-Invariant Artificial Neural Networks) SIANN)" [11].

Researc's method of identifying the type and content of oil and gas reservoir space based on convolutional neural network technology sequentially includes the following detailed steps.
Step 1. Get pictures. Take cores from different types of rocks for sample preparation, use ordinary thin slices, cast thin slices and scanning electron microscopy to observe the samples and obtain images. The sample is artificially identified to identify the type and content of pores.

Step 2. Design the convolutional neural network structure. The complete convolutional neural network includes. input layer, convolution layer, pooling layer, fully connected layer, output layer. The above structure can only classify the type of reservoir space and cannot identify its content. Therefore, a softmax layer is added to the conventional structure to ensure accurate output of different reservoir space types and their contents.

Step 3. Input the image. Input the extracted original rock image and let the convolutional neural network directly train it.

Step 4. Convolution. Convolution extracts rock features, and uses the non-linear mapping of the activation function to obtain a new feature map. The activation function here can be sigmoid, tanh or relu function.

Step 5. pooling. Using the method of extracting the maximum or average value of the feature value, the main feature information of the image is retained, and the amount of calculation is further reduced.

Step 6. Iteration of convolution and pooling. Repeat convolution and pooling to further extract the most important feature information and reduce the amount of calculation.

Step 7. The output of the fully connected layer and the softmax layer. After fully extracting the features, the feature vector is input to the fully connected layer, and the type and content of the reservoir space are output through the softmax layer.

Step 8. Calculate the error. Calculate the error between the actual output and the expected output. If the accuracy requirements are met, the training can be stopped. Otherwise, return to step 3.

\section{3 application effect}

The Ordos Basin is located in the central part of the Chinese mainland, with an area of $25 \times 10^{4} \mathrm{~km}^{2}$. It is the second largest sedimentary basin on land in China and is rich in oil, natural gas and coal resources. The Ordos Basin was originally a part of the North China Plate, which decomposed from the North China Plate after the Indosinian movement and gradually evolved into a large craton basin with a rectangular outline[12,13]. The North China platform, called the Sino-Korean quasi platform by Huang Jiqing, includes the entire North China north of the Qinling Mountains, the southern northeast, the Bohai Sea, the North Yellow Sea, and the northern part of North Korea. It is generally triangular in shape and is separated from adjacent units by deep faults[14,15]. The current structural form of the basin is a north-south rectangular basin, and the basin presents a large asymmetric syncline with a gentle east flank and a steep and narrow west flank. The current Ordos Basin has a simple internal structure and a gentle structure, generally 
less than $1^{\circ}$. The basin has developed a series of lowamplitude nose-like structures formed by differential compaction, which are arranged in an east-west direction, creating good trap conditions for the formation of structural-lithological oil reservoirs [16,17]. According to the evolution history of the basin and the current structural morphology, the Ordos Basin is divided into six structures including the flexure belt in the west of Shanxi, the Yishan (Northern Shaanxi) slope, the Tianhuan depression, the western margin thrust belt, the Yimeng uplift, and the Weibei uplift.

According to outcrop, drilling and seismic data, the area has developed huge Paleozoic and Mesozoic strata, and the Cenozoic is very thin. Among them, the Silurian, Devonian, Upper Jurassic, Upper Cretaceous and Lower Tertiary are all missing. The surface is mostly covered by Quaternary loess. The Mesozoic is composed of a single river-lacustrine facies coal-bearing sedimentary formation, especially the Upper Triassic lacustrine facies deposition has the largest thickness and distribution range, and is one of the most important oil and gas enrichment horizons in the Ordos Basin. The Upper Triassic Yanchang Formation is mainly composed of light gray, brown gray medium-fine sandstone, dark gray siltstone, gray-black mudstone with black carbonaceous mudstone, shale and oil shale, and occasionally thin tuffaceous mudstone, coal line or thin coal seam . It is in parallel unconformity contact with the underlying Middle Triassic Zhifang Formation and the overlying Lower Jurassic Fuxian Formation. Generally, the Yanchang Formation is divided into five lithological assemblages. For the needs of production and research in the oil field, the Yanchang Formation is subdivided into 10 lithological sections, of which the Chang 6 section is the research section.

Reconstructed by sedimentation, diagenesis, etc., the reservoir of the Chang 6 member in the study area is tight and its physical properties are poor. The porosity is usually $6 \% \sim 14 \%$, and the air permeability is less than $1 \times 10^{-3} \mu \mathrm{m}^{2}$. The lithology is mainly fine sandstone and siltstone. The rock types are mainly lithic feldspar sandstone and feldspar lithic sandstone, followed by lithic sandstone and feldspar sandstone. The pore types are mainly dissolved pores or intergranular poresdissolved pores. The throat is small, the average median radius is only $0.14 \mu \mathrm{m}$, and the average displacement pressure is $2.08 \mathrm{MPa}$. The composition and content of clay minerals and other cements also vary greatly, resulting in significant differences in pore structure characteristics and reservoir physical properties in different zones, with strong heterogeneity.

Taking the Chang 6 oil layer group in a block of the Ordos Basin as an example, according to the petrological characteristics of the reservoir, through the analysis and statistics of 100 rock thin slices and 35 scanning electron microscope samples, the reservoir space of the Chang 6 oil layer group in the study area is identified manually. Intergranular pores and intragranular dissolved pores are dominant ( figure 1) .Intergranular pores When particles accumulate, the pore space formed by the particles supporting each other. The degree of development of intergranular pores is closely related to factors such as particle content, size, sortability, arrangement and filling content. The retention of residual primary intergranular pores in the reservoir is a key factor in determining the quality of the reservoir. Intragranular dissolution pores refer to the pores formed by partial dissolution of various carbonate particles due to selective dissolution. When the dissolution spreads to the entire particle to form pores with the same shape and size as the original particle, it is also It can be called mold porosity.

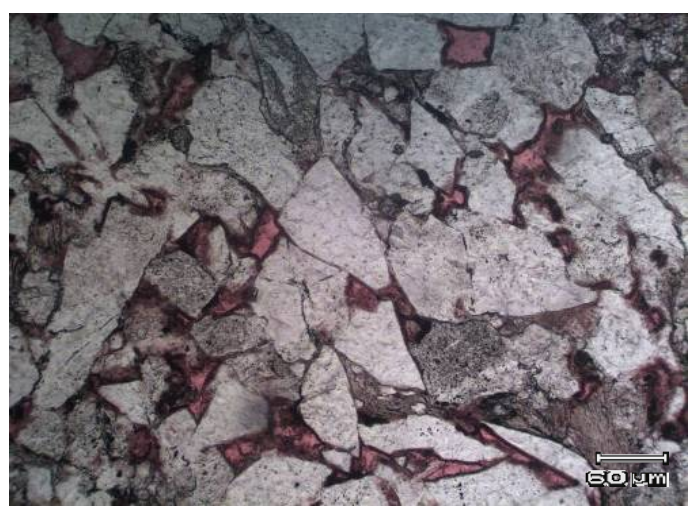

a. Well A, Chang 6, residual intergranular pores, single polarized light, cast body piece

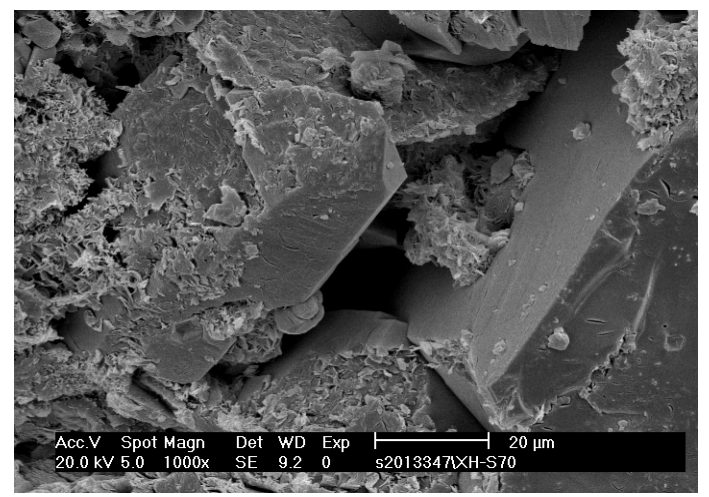

b. Well B, Chang 6, residual intergranular pores, SEM

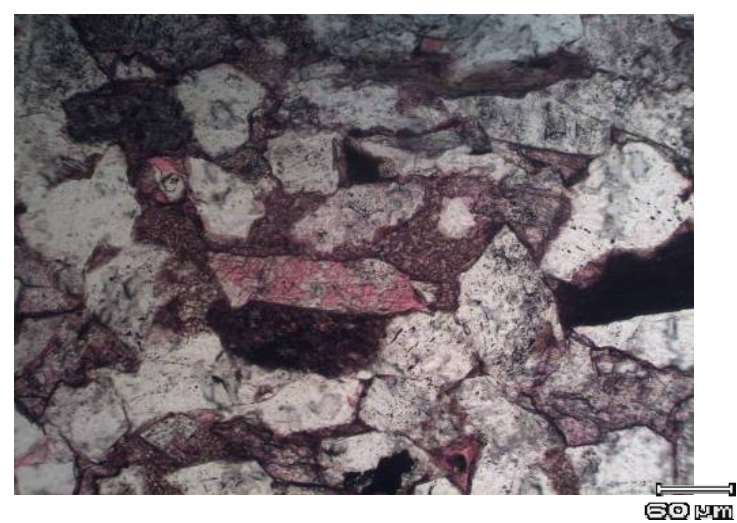

c. Well C, Chang 6, intragranular dissolved pores and residual intergranular pores, single polarized light, cast body sheet 


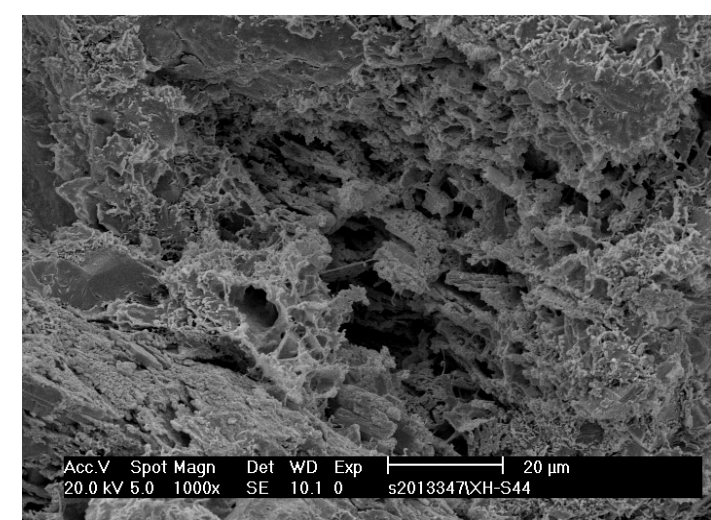

d. Well D, Chang 6, dissolved pores in the grain, SEM

Figure 1 Microphotograph of the reservoir space in the study area

The absolute content of intergranular pores and intragranular dissolved pores were $0.731 \%$ and $0.951 \%$, respectively. Using convolutional neural network-based oil and gas reservoir space technology for identification, the average error of the two pores is $20 \%$. The accuracy meets the requirements (Table 1).

Table 1 Absolute content statistics of main reservoir space types in the study area

\begin{tabular}{|c|c|c|c|c|}
\hline \multicolumn{2}{|c|}{ Manual recognition results } & \multicolumn{2}{|c|}{$\begin{array}{c}\text { Convolutional neural } \\
\text { network recognition results }\end{array}$} & \multirow{2}{*}{$\begin{array}{c}\text { Average } \\
\text { error of } \\
\text { two } \\
\text { pores }\end{array}$} \\
\hline $\begin{array}{l}\text { Intergranular } \\
\text { pores }(\%)\end{array}$ & $\begin{array}{c}\text { Intragranular } \\
\text { Dissolved } \\
\text { pores }(\%)\end{array}$ & $\begin{array}{l}\text { Intergranular } \\
\text { pores }(\%)\end{array}$ & $\begin{array}{c}\text { Intragranular } \\
\text { Dissolved } \\
\text { pores }(\%)\end{array}$ & \\
\hline 0.731 & 0.951 & 0.583 & 0.760 & $20 \%$ \\
\hline
\end{tabular}

\section{Conclusion}

The use of convolutional neural network technology for oil and gas reservoir space identification is not only simple and objective, but also saves labor costs, has high efficiency, and has high accuracy.However, the excellent performance of convolutional neural networks is limited by the self-supervised training process and the largescale data set to provide sufficient training samples. Especially with the rapid development of information technology and computer software and hardware technology, if more raw data is quickly accumulated in production and life, sample acquisition work such as data labeling, sorting and cleaning will greatly increase production costs. Correspondingly, the convolutional neural network also needs more layer structure and training parameters, which undoubtedly increases the calculation amount of the network, prolongs the training time, and affects the performance of the convolutional neural network. In addition, how to analyze the latent semantic attributes of the image itself more clearly and express the actual meaning of the image more clearly will also be the next direction of work.

\section{References}

1. Li Zhuowen. A comparative study on the difference of shale oil and gas reservoir space and their modes of occurrence[D]. Yangtze University, 2016.
2. Li Zhuowen, Pan Renfang, Shao Yan, Yang Baogang. A comparative study of shale oil and gas reservoir spatial differences and occurrence modes[J]. Journal of Chongqing University of Science and Technology (Natural Science Edition), 2015, 17(05):1-4.

3. Zhao Chenglin. Formation mechanism of volcanic rock reservoir and its oil and gas content[J].Geological Review,1996,42(S1):37-43.

4. Jiu Bo, Huang Wenhui, He Mingqian, etc. A method based on Matlab to analyze the pore characterization of tight sandstone reservoirs [P]. Chinese Patent: CN108956416A, 2018-12-07

5. Qi Minghui, Li Junjun, Cao Qian. Study on the characterization of shale pore structure based on scanning electron microscope and JMicroVision image analysis software[J].Rock and Mineral Testing, 2019,38(03):260-269.

6. Li Wenhou, Pang Jungang, Cao Hongxia, etc. Sedimentary system and lithofacies paleogeographic evolution of the Late Triassic Yanchang period in Ordos Basin[J]. Journal of Northwest University (Natural Science Edition),2009 ,39(03):501 -506.

7. Li Haiyan, Yue Dali, Zhang Xiujuan. Micro-pore structure characteristics and classification and evaluation methods of low-permeability reservoirs in Sulige Gas Field[J]Earth Science Frontiers, 2012, 19(2): 133-140.

8. Li Tianyuan. Evaluation of characteristics of Chang 7 source rock in Zhidan area, Ordos Basin[J]. Unconventional Oil \& Gas,2017,4(1):23 29.

9. Goodfellow, I., Bengio, Y., Courville, A.. Deep learning (Vol. 1). Cambridge: MIT press, 2016: 326366

10. Gu, J., Wang, Z., Kuen, J., Ma, L., Shahroudy, A., Shuai, B., Liu, T., Wang, X., Wang, L., Wang, G. and Cai, J., 2015. Recent advances in convolutional neural networks. arXiv preprint arXiv:1512.07108.

11. Zhang, W., 1988. Shift-invariant pattern recognition neural network and its optical architecture. In Proceedings of annual conference of the Japan Society of Applied Physics.

12. Zhang Kang. 1989. Ordos fault block structure and resources[M]. Xi'an: Shaanxi Science and Technology Press.

13. Song Guochu, Luo Shaolun, Zhang Shifu, etc. 1993. Geological laws and exploration experience of Mesozoic continental oilfields in Shaan-Gan-Ning Basin. Changqing Research Institute report, internal data.

14. Yang Junjie. Tectonic evolution and oil and gas distribution in the Ordos Basin[M]. Beijing: Petroleum Industry Press, 2002

15. He Zixin. Ordos Basin Evolution and Oil and Gas[M]. Beijing: Petroleum Industry Press, 2003 